\title{
Estudo do tratamento de oxidação em diferentes microestruturas da liga de titânio
}

\author{
Fabricia Assis Resende Gonçalves ${ }^{1}$ \\ Renata Jesuina Takahash ${ }^{2}$ \\ Danieli Aparecida Pereira Reis ${ }^{3}$ (C)
}

\section{Resumo}

A liga Ti-6Al-4V é a liga de titânio mais usada em aplicações estruturais de engenharia pela combinação de resistência mecânica em altas temperaturas, baixa massa específica e resistência à corrosão. Contudo, uma das limitações da liga é a afinidade do titânio por oxigênio a partir de $600^{\circ} \mathrm{C}$, tornando a liga susceptível à oxidação. Portanto, neste trabalho se estudou a oxidação na liga de titânio (Ti-6Al-4V) com diferentes microestruturas: equiaxial, Widmanstätten, martensítica e bimodal obtidas por tratamento térmico. As amostras com as diferentes microestruturas foram submetidas a tratamento térmico de oxidação nas temperaturas de 500, 600, 700 e $800{ }^{\circ} \mathrm{C}$ por 48 horas ao ar. Os resultados mostraram que as amostras oxidadas formaram uma película de óxido sobre a superfície que foram caracterizadas por microscopia óptica e microscopia eletrônica de varredura, por medição da variação da massa das amostras e por análise de microdureza. A microestrutura Widmanstätten apresentou-se mais resistente à oxidação pela formação de grãos mais grosseiros e formação de película de óxidos mais espessos e mais resistentes a deformação localizada.

Palavras-chave: Ti-6Al-4V; Oxidação; Tratamento Térmico.

\section{Study of the treatment of oxidation in different microstructures of the titanium alloy}

\begin{abstract}
The Ti-6Al-4V alloy is the most commonly used titanium alloy in structural engineering applications by combining mechanical strength at high temperatures, low specific mass, and corrosion resistance. However, one of the limitations of the alloy is the affinity of titanium for oxygen from $600{ }^{\circ} \mathrm{C}$, making the alloy susceptible to oxidation. The purpose of this work was to study the oxidation of titanium alloys (Ti-6Al-4V) with different microstructures: equiaxial, Widmanstätten, martensite, and bimodal, which were formed from heat treatment. The samples with different microstructures were subjected to oxidation heat treatment at temperatures of $500,600,700$, and $800^{\circ} \mathrm{C}$ for 48 hours in the air. The oxidized samples formed an oxide film on the surface, which was characterized by optical and scanning electron microscopy, by measuring the mass of the samples and by microhardness analysis. The Widmanstätten microstructure was more resistant to oxidation by the formation of coarser grains and the formation of thicker oxide films and more resistant to localized deformation.
\end{abstract}

Keywords: Titanium; Ti-6Al-4V; Oxidation; Heat Treatment.

\section{Introdução}

A liga Ti-6Al-4V é a mais importante das ligas de titânio usadas em engenharia combinando propriedades atrativas com trabalhabilidade. Esta liga tem sido muito utilizada na indústria aeronáutica e aeroespacial, particularmente para aplicações que requerem resistência em alta temperatura [1]. Assim, para se projetar novas ligas de titânio com melhores propriedades e para otimizar as condições de processamento de forjamento à quente da liga Ti-6Al-4V é necessário entender os mecanismos associados à deformação sob temperaturas elevadas [2-10].

Em altas temperaturas, a resistência mecânica específica das ligas de titânio é particularmente atraente e há notáveis avanços no desenvolvimento de ligas de titânio com alta resistência à tração, ductilidade e resistência à fluência em

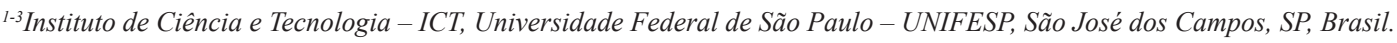

*Autor correspondente:: fabricia.resende@unifesp.br 
temperaturas elevadas. Contudo, as ligas como materiais estruturais a temperatura máxima de aplicação limita-se pelo comportamento de oxidação resultado da afinidade do titânio por oxigênio [3-11].

$\mathrm{O}$ oxigênio absorvido combina-se quimicamente com o titânio e outros elementos de ligas para formar uma camada de óxido na superfície, condicionando a liga para um ganho de massa quando exposta a temperaturas superiores a $600^{\circ} \mathrm{C}$. Nesta condição a difusão intersticial do oxigênio no metal torna-se ativo [4-12].

Estudos recentes de difusão de oxigênio podem ser correlacionados com o tamanho de grão e microestrutura do material. A elevação dos níveis de oxigênio é associada com o aumento da microdureza e da diminuição da ductibilidade do titânio [5-13].

As propriedades mecânicas destas ligas são muito sensíveis à microestrutura, que geralmente consiste em rearranjo de duas fases $(\alpha+\beta)$, que variam amplamente dependendo do processamento e do tratamento térmico realizado [6].

Por fim, dentre as modificações microestruturais por tratamento térmico das ligas de titânio podem-se citar as microestruturas equiaxial, Widmanstätten, bimodal e martensítica [8-17]. É comum encontrar diversos relatados na literatura apresentando as correlações entre microestrutura e propriedades mecânicas em altas temperaturas [8-20]. Neste contexto, o presente trabalho tem como objetivo o estudo da microestrutura da liga de titânio que é mais resistente a oxidação nas temperaturas de $500,600,700$ e $800^{\circ} \mathrm{C}$ a partir destas microestrutura avaliada por análise microestrutural, microdureza e variação de ganho de massa da liga.

\section{Materiais E Métodos}

A liga Ti-6Al-4V foi adquirida junto à Empresa Multialloy Eng. Mat. Ltda. Ela é obtida na forma de barras cilíndricas na condição forjada e recozida a $190{ }^{\circ} \mathrm{C}$ durante 6 horas e resfriada ao ar, apresentando a microestrutura equiaxial.

A análise química foi realizada via espectroscopia de emissão óptica com plasma indutivamente acoplado (ARL, modelo 3410). A Tabela 1 apresenta os dados da análise da composição química.

Os corpos de prova em forma de pastilhas de 3,6 mm de diâmetro foram cortados em cortadeira Isomet na dimensão de $2 \mathrm{~mm}$ de espessura. $\mathrm{O}$ corpo de prova da liga de titânio com a microestrutura equiaxial foi submetida a três condições de tratamentos térmicos visando a obtenção das microestruturas Widmanstätten, martensita e bimodal.

Em seguida, as amostras foram submetidas ao tratamento de oxidação em diferentes temperaturas $(500,600,700 \mathrm{e}$ $800^{\circ} \mathrm{C}$ ) por 48 horas. Estes tratamentos são mencionados brevemente abaixo.

Tabela 1. Composição química (\% em peso) da liga Ti-6Al-4V

\begin{tabular}{cccc}
\hline Elemento & Ti & Al & V \\
\hline \% em peso & 89,16 & 6,61 & 4,23 \\
\hline
\end{tabular}

\subsection{Tratamento térmico para obtenção da estrutura Widmanstätten}

Os corpos de prova foram encapsulados em tubos de quartzo sob vácuo para o tratamento térmico no forno (Lindberg/Blue) aquecido até o patamar de $1050{ }^{\circ} \mathrm{C}$ durante 30 minutos. O resfriamento ocorreu dentro do forno até a temperatura ambiente na taxa de $6{ }^{\circ} \mathrm{C}$ por minuto. Após o tratamento térmico, os corpos de prova foram lavados em solução de decapagem de $\mathrm{HF}-0,2 \mathrm{~mL} / \mathrm{HNO}_{3}-2 \mathrm{~mL} / \mathrm{H}_{2} \mathrm{O}-30 \mathrm{~mL}$.

\subsection{Tratamento térmico para obtenção da estrutura martensítica}

Os corpos de prova foram encapsulados em tubos de quartzo sob vácuo para o tratamento térmico no forno (Lindberg/Blue) aquecido até o patamar de $1050{ }^{\circ} \mathrm{C}$ durante 60 minutos. Após o tratamento térmico os corpos de prova foram imergidos imediatamente em água na temperatura ambiente e, posteriormente, lavados em solução de decapagem de $\mathrm{HF}-0,2 \mathrm{~mL} / \mathrm{HNO}_{3}-2 \mathrm{~mL} / \mathrm{H}_{2} \mathrm{O}-30 \mathrm{~mL}$.

\subsection{Tratamento térmico para obtenção da estrutura bimodal}

O tratamento térmico foi realizado em duas etapas. A primeira etapa ocorreu no forno (Lindberg/Blue) aquecido a $950{ }^{\circ} \mathrm{C}$ por 1 hora. Após esta etapa, o corpo de prova foi resfriado em água na temperatura ambiente, seguido de lavagem de decapagem de $\mathrm{HF}-0,2 \mathrm{~mL} / \mathrm{HNO}_{3}-2 \mathrm{~mL} / \mathrm{H}_{2} \mathrm{O}-30 \mathrm{~mL}$. $\mathrm{Na}$ segunda etapa, os corpos de prova foram encapsulados em tubo de quartzo em atmosfera inerte e submetidos ao forno (Lindberg/Blue) a $600^{\circ} \mathrm{C}$ por 24 horas. O resfriamento ocorreu ao ar até a temperatura ambiente.

\subsection{Tratamento de oxidação}

O tratamento de oxidação foi realizado em quatro amostras com microestrutura equiaxial, bimodal, martensita e Widmanstätten, de modo que a superfície das amostras foi preparada seguindo os padrões usuais de metalográfia. Em outras palavras, o procedimento consistiu no embutimento a quente $\left(150^{\circ} \mathrm{C}\right)$ sob pressão de $21 \mathrm{MPa}$, seguido do lixamento utilizando-se uma politriz da marca Panambra com lixas a base de $\mathrm{SiC}$ na sequência de 600, 1200 e 2400 de granulometria. $\mathrm{Na}$ etapa de polimento das amostras utilizou-se o pano para polimento OP-Chem com uma solução de polimento a base de sílica coloidal $(0,06 \mu \mathrm{m})$ e $\mathrm{H}_{2} \mathrm{O}_{2}$. Além disso, utilizou-se um forno da marca Fornitec em atmosfera ao ar por 48 horas nas temperaturas de 500,600, 700, $800^{\circ} \mathrm{C}$.

Para encontrar a variação da massa nas amostras (crescimento das camadas de óxido) utilizou-se a balança analítica para medição das massas das amostras antes e depois dos tratamentos térmicos de oxidação.

A caracterização microestrutural ocorreu via microscopia óptica (Weiss, Discovery V8) e microscopia 
eletrônica de varredura (LEO, 435VPI) e a análise de microdureza Vickers foi realizada utilizando-se uma carga de $300 \mathrm{kgf}$ em equipamento Future Tech, modelo 7. As microindentações para obtenção dos valores de microdureza foram realizadas na camada de óxido formada pós tratamento de oxidação.

\section{Resultados}

A condição como recebida da liga Ti-6Al-4V apresentando a microestrutura equiaxial foi submetida aos tratamentos térmicos que promoveram a alteração para as microestruturas Widmanstätten, bimodal e martensítica, como são apresentadas na Figura 1.

As amostras foram submetidas aos tratamentos de oxidação a $500,600,700,800^{\circ} \mathrm{C}$ por 48 horas e as microestruturas resultantes a partir das microestruturas equiaxial, Widmanstätten, martensítica e bimodal são apresentadas nas Figuras 2, 3, 4 e 5, respectivamente.

As amostras apresentaram variação em sua massa com o tratamento térmico de oxidação conforme apresentado na Figura 6.

$\mathrm{O}$ resultado da variação de massa referiu-se a formação e o crescimento de uma película de óxido sobre a superfície com o aumento da temperatura do tratamento de oxidação.

A camada de óxido formada foi avaliada pela análise de microdureza apresentada na Figura 7.

\section{Discussão}

A partir da condição inicial do corpo de prova da liga de titânio com estrutura equiaxial, observou-se a presença de duas fases na Figura 1-a. Estas fases correspondem a fase $\alpha$ (hexagonal compacta) que se identifica na região mais clara, e fase $\beta$ (cúbica de corpo centrado), na região mais escura.

Os tratamentos térmicos que objetivaram a mudança microestrutural Widmanstätten, martensita e bimodal ocorreram satisfatoriamente, de modo que as características peculiares de cada microestrutura foram identificadas nos painés b, c e d da Figura 1, respectivamente.

A microestrutura Widmanstätten apresentou a estrutura lamelar com regiões mais claras (fase $\alpha$ ) os contornos mais escuros (fase $\beta$ ), com formatação clássica de bastonetes, conhecida como basketwave. A microestrutura martensítica caracterizou-se pela forma acicular (formato de agulhas), onde a fase $\alpha$ se forma a partir dos contornos de grãos $\beta$. E a microestrutura bimodal resultante consistiu em martensita temperada nas regiões de tonalidade mais escura e, em fase $\alpha$ nas regiões de tonalidades mais claras, juntamente com uma pequena concentração de fase $\beta$ residual.

Após o tratamento t de oxidação, observou-se alterações nas microestruturas das amostras. Além disso, foi identificado um ganho de massa e um aumento de dureza superficial com o aumento da temperatura de tratamento térmico.

Na comparação das microestruturas, observou-se os grãos mais refinados para a microestrutura equiaxial e mais

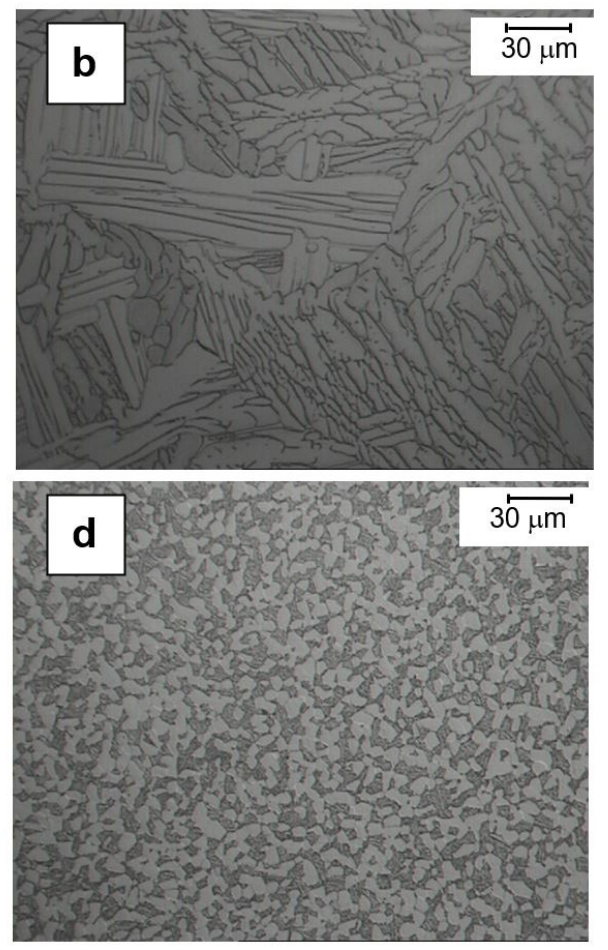

Figura 1. Micrografias da liga Ti-6Al-4V via microscopia óptica. a) Microestrutura equiaxial [19]. b) Microestrutura Widmanstätten [19]. c) Microestrutura martensítica [19]. d) Microestrutura bimodal [19]. 

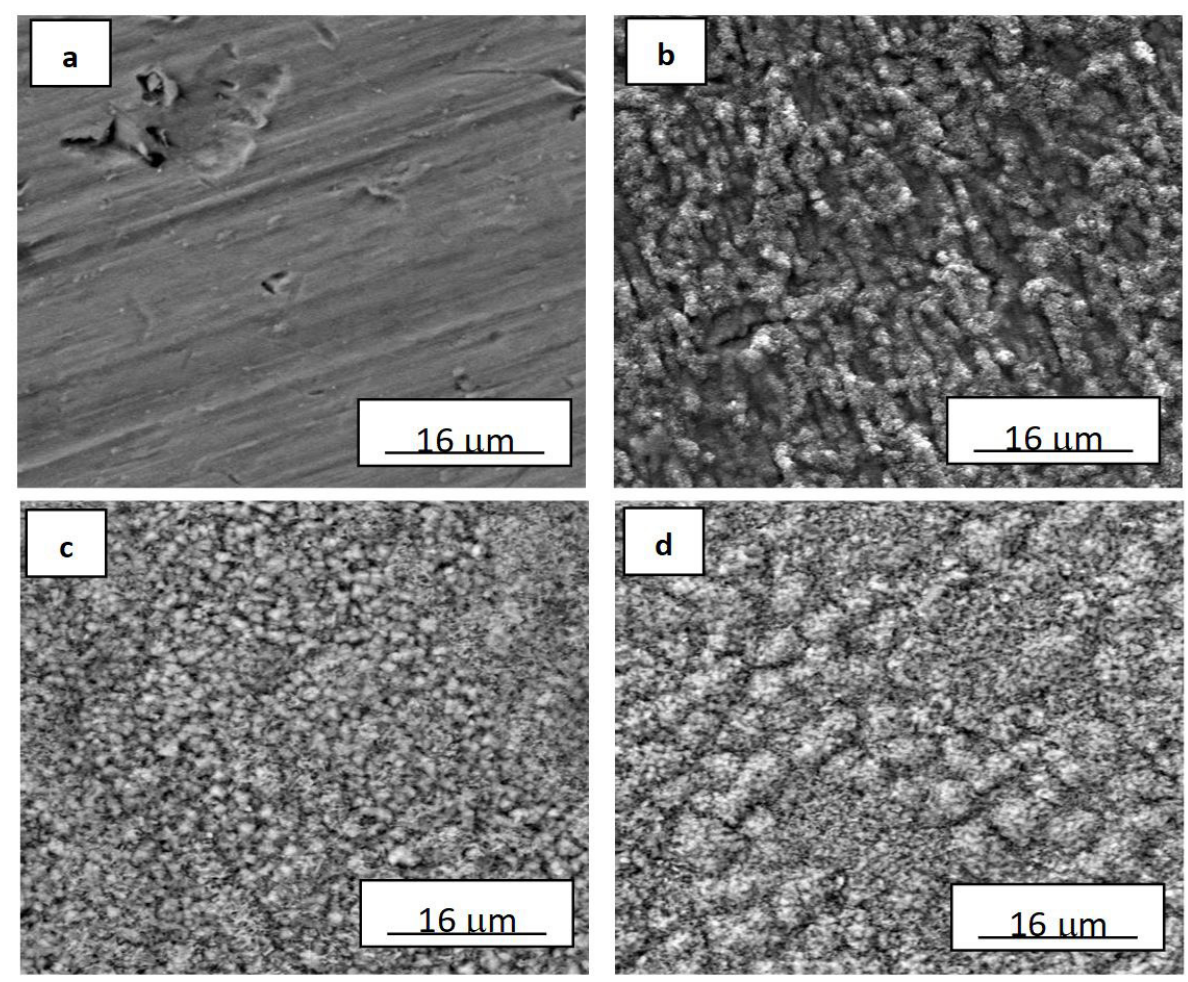

Figura 2. Microestrutura equiaxial da liga de titânio após tratamento de oxidação por 48 horas via $\mathrm{MEV}$. a) $500^{\circ} \mathrm{C}$, b) $600^{\circ} \mathrm{C}$, c) $700^{\circ} \mathrm{C}$ e d) $800^{\circ} \mathrm{C}$.
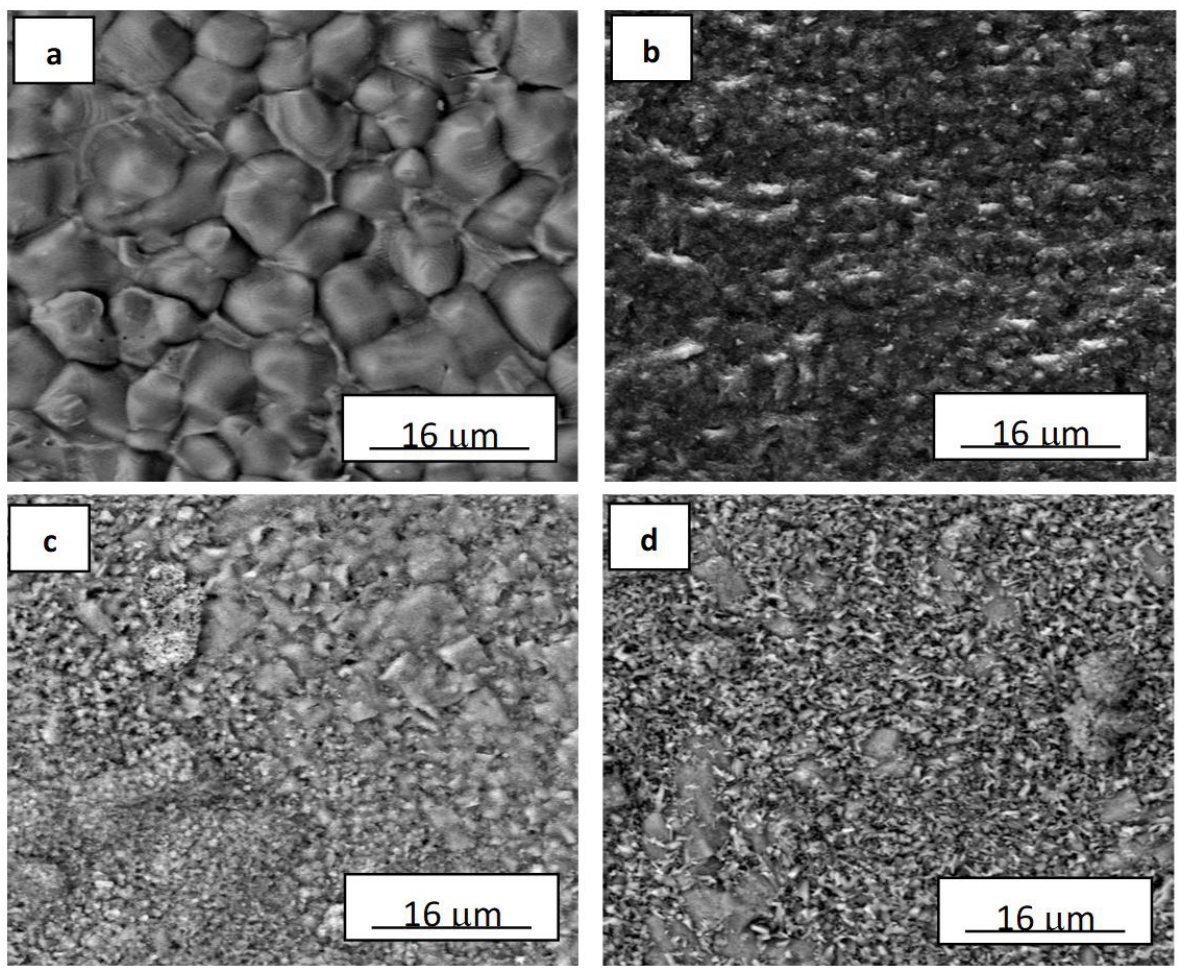

Figura 3. Microestrutura Widmanstätten da liga de titânio após tratamento de oxidação por 48 horas via $\mathrm{MEV}$. a) $\left.\left.500^{\circ} \mathrm{C}, \mathrm{b}\right) 600^{\circ} \mathrm{C}, \mathrm{c}\right) 700^{\circ} \mathrm{C}$ e d) $800^{\circ} \mathrm{C}$.

grosseiros para a microestrutura Widmanstätten. Este fato pode ocasionar uma maior resistência à oxidação da liga, pois quanto maior o tamanho de grão menor será a energia de ativação presente no contorno de grão e com isso o material reagirá menos com o meio [8].
Observou-se que a modificação superficial para todas as microestruturas foi ocasionada pela formação e crescimento da camada de óxido. Esta película de óxido aumentou o valor de ganho de massa das amostras com o aumento da temperatura, sendo que a partir de $700{ }^{\circ} \mathrm{C}$, 

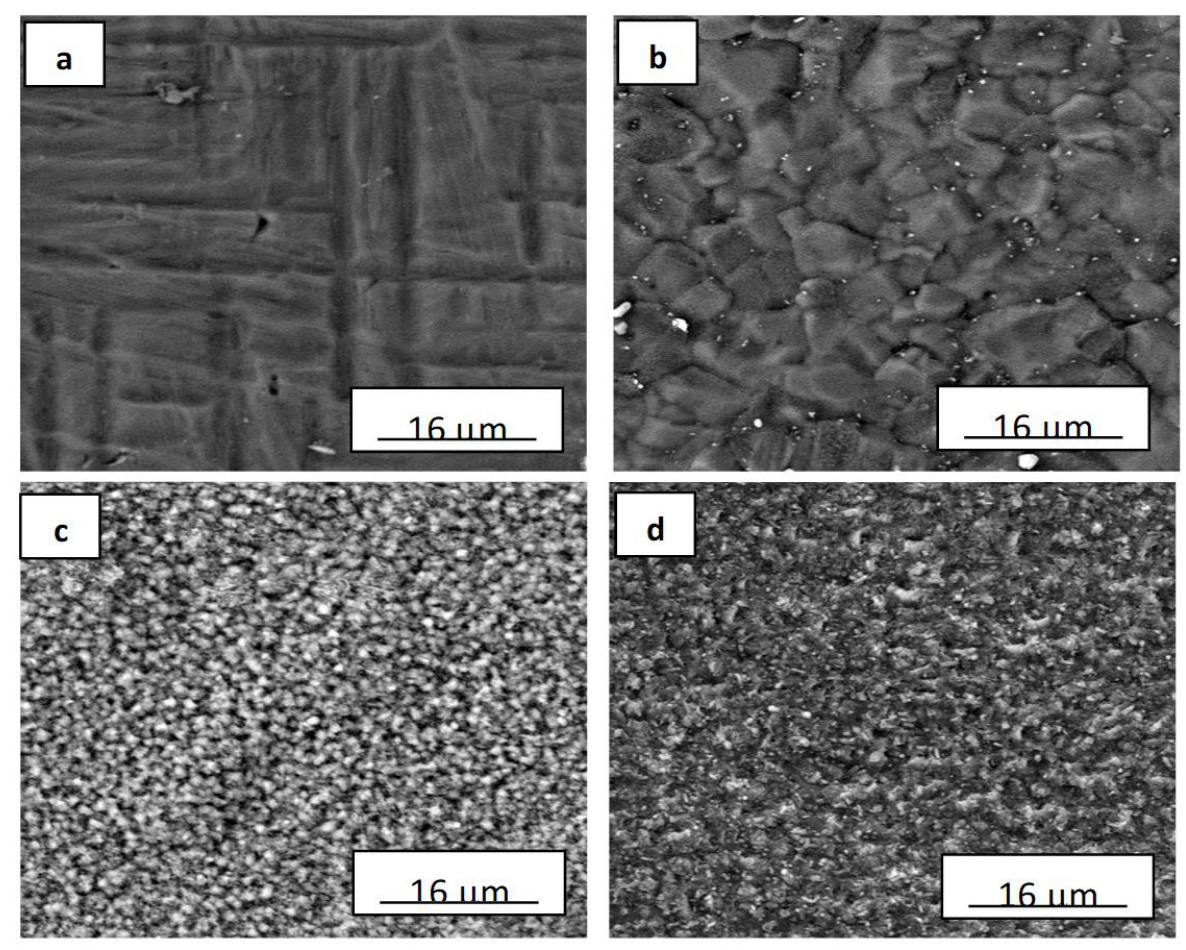

Figura 4. Microestrutura martensita da liga de titânio após tratamento de oxidação por 48 horas via MEV. a) $500^{\circ} \mathrm{C}$, b) $600^{\circ} \mathrm{C}$, c) $700^{\circ} \mathrm{C}$ e d) $800^{\circ} \mathrm{C}$.
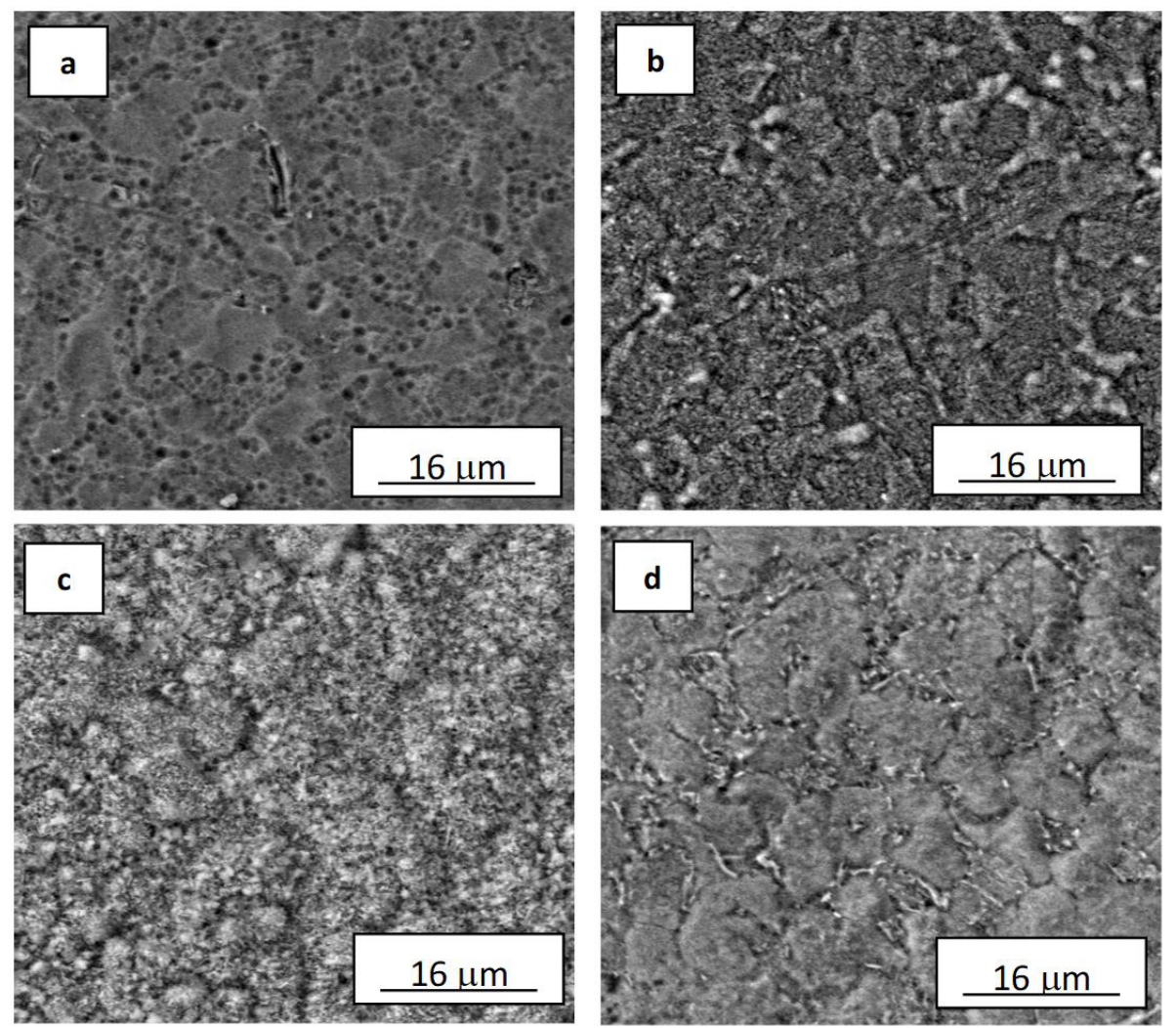

Figura 5. Microestrutura bimodal da liga de titânio após tratamento de oxidação por 48 horas via MEV. a) $500^{\circ} \mathrm{C}$, b) $\left.600^{\circ} \mathrm{C}, \mathrm{c}\right) 700^{\circ} \mathrm{C}$ e d) $800^{\circ} \mathrm{C}$.

o aumento do ganho de massa seguiu um comportamento exponencial, conforme indicado na Figura 6. A comparação do ganho de massa entre as microestruturas indicou que não houve uma diferença significativa entre elas e que todas as amostras apresentam uma tendência de crescimento da formação de óxido com aumento da temperatura.

A formação de óxidos na superfície foi devido à difusão do oxigênio, já que o nitrogênio e outras espécies são menos 


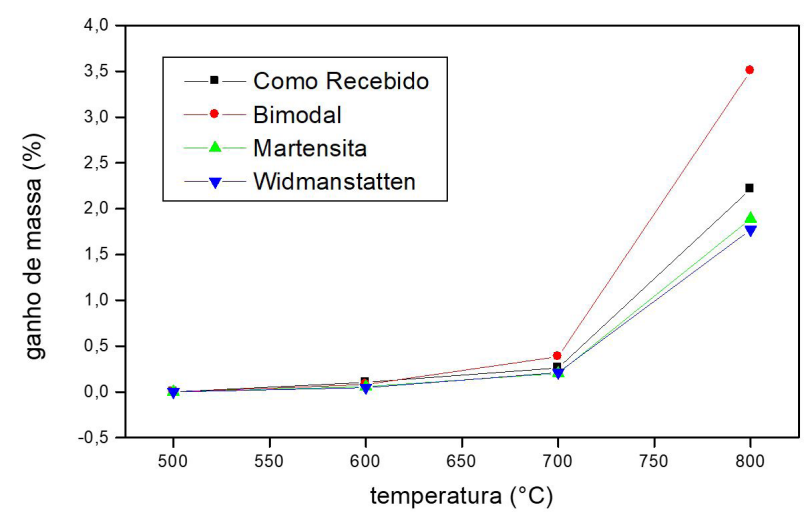

Figura 6. Medidas da massa da liga titânio em função das microestruturas e das temperaturas do tratamento de oxidação por 48 horas.

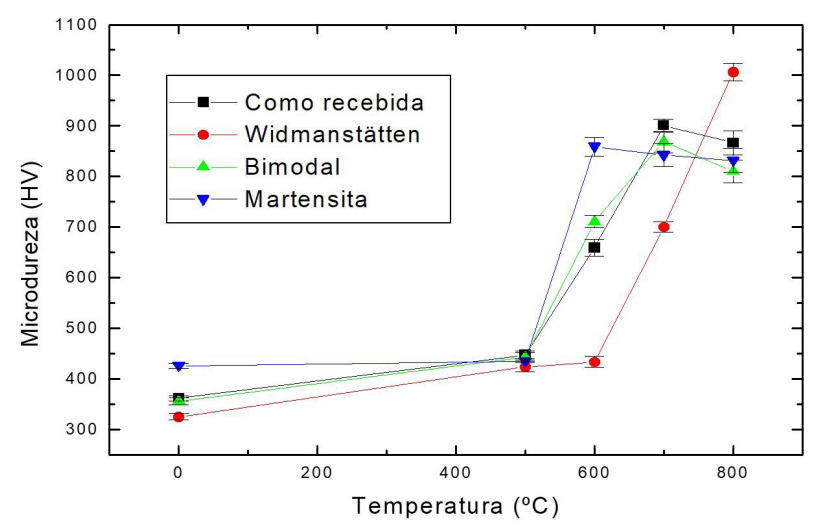

Figura 7. Medidas de microdureza Vickers da liga titânio em função das microestruturas e das temperaturas do tratamento de oxidação por 48 horas.

reativos nas condições de tratamento deste trabalho [20]. $\mathrm{O}$ oxigênio absorvido combinou-se quimicamente com o titânio e outros elementos da liga para formar uma camada de óxido, além da difusão intersticial no metal [8]. Quando exposto ao ar em temperaturas elevadas acima de $300^{\circ} \mathrm{C}$, o crescimento da camada de óxido e a difusão de oxigênio no metal ocorrem simultaneamente [20]. Ademais, com o aumento da temperatura de tratamento de oxidação, a aceleração da taxa de oxidação permitiu a formação de uma camada mais espessa de óxido e zona de difusão de oxigênio mais profunda.

O oxigênio difundido na liga induz um aumento da dureza e a quantidade de oxigênio pode ser proporcional à dureza no titânio [21-22], que se confirmou pela análise de microdureza apresentada pela Figura 7.
Experimentos com amostras de liga de titânio submetidas ao tratamento isotérmico de oxidação quando analisadas em seção transversal, gerou um perfil de dureza em função da difusão do oxigênio pela superfície, o que resultou em maiores valores de dureza nas regiões enriquecidas com oxigênio [20].

Na temperatura de $800^{\circ} \mathrm{C}$ observou-se um valor mais elevado de dureza em função da camada de óxido formado, indicando um aumento de resistência a oxidação da liga Ti-6Al-4V com a microestrutura Widmanstätten.

Pela observação das microestruturas, dos valores de ganho de massa e da microdureza, a microestrutura Widmanstätten apresentou-se como a microestrutura mais resistente à oxidação em relação as demais microestruturas.

\section{Conclusão}

Pelo estudo da oxidação da liga de titânio a partir das microestruturas equiaxial, Widmanstätten, martensítica e bimodal conclui-se que:

- Os tratamentos de oxidação das amostras promoveram a formação e crescimento da camada de óxido sobre a superfície, resultando em ganho de massa.

- O ganho de massa foi crescente com o aumento da temperatura para todas as microestruturas.

- O aumento da dureza superficial foi resultado da formação de películas mais espessas e aderentes que tornaram a liga mais resistente a deformação plástica localizada. Este fato ocorreu devido a ação do oxigênio na liga de titânio pela atmosfera oxidante com o aumento da temperatura.

- A microestrutura Widmanstätten foi a mais resistente a oxidação a $800^{\circ} \mathrm{C}$ da amostra. Os grãos formados foram grosseiros e a formação de película de óxido protetora obteve maior dureza, o que sugere um aumento da resistência a oxidação.

\section{Agradecimentos}

O presente trabalho foi realizado com apoio da Coordenação de Aperfeiçoamento de Pessoal de Nível Superior - Brasil (CAPES) - Código de Financiamento 001.

\section{Referências}

1 Leyens C, Peters M. Titanium and titanium alloys. 3rd ed. Weinheim: Wiley; 2003.

2 Zhecheva A, Sha W, Malinov S, Long A. Enhancing the microstructure and propertiesof titanium alloys through nitriding and other surface engineering methods. Surface and Coatings Technology. 2005;200:2192-2207.

3 Reis AG, Reis DAP, Moura Neto C, Barboza MJR, Oñoro J. Creep behavior and surface characterization of a laser surface nitrided Ti-6Al-4V alloy. Materials Science and Engineering A. 2013;577:48-53. 
4 Oliveira AC, Oliveira RM, Reis DAP, Carreri FC. Effect of nitrogen high temperature plasma based ion implantation on the creep behavior of Ti-6Al-4V alloy. Applied Surface Science. 2014;311:239-244.

5 Reis DAP, Reis AG, Yogi LM, Silva MM, Ueda M, Zepka S. Comparation between Laser Surface Nitriding and Nitrogen Plasma Immersion Ion Implantation (N-PIII) on Creep Behavior of Ti-6Al-4V Alloy. Materials Science Forum. 2014;802:462-466.

6 Briguente LANS, Briguente FP, Reis DAP, Moura Neto C, Resende FA. Avaliação do comportamento em fluência da liga Ti-6Al-4V com estrutura martensítica e equiaxial a $600^{\circ} \mathrm{C}$. Tecnologia em Metalurgia. Materiais e Mineração (Impresso). 2013;10:324-328.

7 Sugahara T, Martins GV, Montoro FE, Merij Neto A, Massi M, da Silva Sobrinho AS, et al. Creep behavior evaluation and characterization of $\mathrm{SiC}$ film with $\mathrm{Cr}$ interlayer deposited by HiPIMS in Ti-6Al-4V alloy. Surface and Coatings Technology. 2017;309:410-416.

8 Sugahara T, Reis DAP, Moura Neto C, Barboza MJR, Perez EAC, Piorino Neto F, et al. The effect of Widmanstätten and equiaxed microestructures of Ti-6Al-4V on the oxidation rate and creep behavior. Materials Science Forum. 2010;636/637:657-662.

9 Reis DAP, Silva CRM, Nono MCA, Barboza MJR, Piorino Neto F, Perez EAC. Plasma-Sprayed Coatings for Oxidation Protection on Creep of the Ti-6Al-4V Alloy. Materials at High Temperatures. 2005;22:449-452.

10 Reis DAP, Moura Neto C, Silva CRM, Barboza MJR, Piorino Neto F. Effect of coating on the creep behavior of the Ti-6Al-4V alloy. Materials Science and Engineering A. 2008;486:421-426.

11 Donachie MJ. Titanium: A Technical Guide. 2nd ed. Novelty, OH: ASM International; 2000.

12 Froes FH. Titanium: physical metallurgy, processing, and applications. 1st Ed. Novelty, OH: ASM International; 2015.

13 Seco FJ, Irisarri AM. Creep failure mechanisms of a Ti-6Al-4V thick plate. Fatigue \& Fracture of Engineering Materials \& Structures. 2001;24:741-750.

14. American Society of Testing and Materials. Annual Book of ASTM Standards. West Conshohocken: ASTM; 1995.

15 Sahoo R, Jha BB, Sahoo TK. Effect of Microstructure on the Creep Properties of Ti-6Al-4V Alloys: An Analysis. Transactions of the Indian Institute of Metals. 2018;71:1573-1582.

16 Jeng SC. Oxidation behavior and microstructural evolution of hot-dipped aluminum coating on Ti-6Al-4V alloy at $800^{\circ} \mathrm{C}$. Surface and Coatings Technology. 2013;235:867-874.

17 Luan JH, Jiao ZB, Chen G, Liu CT. Improved ductility and oxidation resistance of cast Ti-6Al-4V alloys by microalloying. Journal of Alloys and Compounds. 2014;602:235-240.

18 Lütjering G. Influence of processing on microstructure and mechanical properties of $(\alpha+\beta)$ titanium alloys. Materials Science and Engineering A. 1998;243:32-45.

19 Yogi LM, Sugahara T, Moura Neto C, Reis DAP, Piorino Neto F, Barboza MJR. Efeito do tratamento térmico na fluência da liga Ti-6Al-4V. Revista Brasileira de Aplicações de Vácuo. 2008;27:189-194.

20 Poquillon D, Armand C, Huez J. Oxidation and oxygen diffusion in Ti-6al-4V alloy: Improving measurements during SIMS analysis by rotating the sample. Oxidation of Metals. 2013;79:249-259.

21 Ramachandran M, Mantha D, Williams C, Reddy RG. Oxidation and diffusion in Ti-Al-(Mo, Nb) intermetallics. Metallurgical and Materials Transactions. A, Physical Metallurgy and Materials Science. 2011;42:202-210.

22 Kofstad P. High-temperature oxidation of titanium. Journal of the Less Common Metals. 1967;12:449-464.

Recebido: 10 Jun. 2019

Aceito: 29 Set. 2020 